\title{
On the number of channels required for interference-free wireless mesh networks
}

\author{
Aizaz U Chaudhry*, John W Chinneck and Roshdy HM Hafez
}

\begin{abstract}
We study the problem of achieving maximum network throughput with fairness among the flows at the nodes in a wireless mesh network, given their location and the number of their half-duplex radio interfaces. Our goal is to find the minimum number of non-overlapping frequency channels required to achieve interference-free communication. We use our existing Select $x$ for less than $x$ topology control algorithm (TCA) to build the connectivity graph (CG), which enhances spatial channel reuse to help minimize the number of channels required. We show that the TCA-based CG approach requires fewer channels than the classical approach of building the CG based on the maximum power. We use multi-path routing to achieve the maximum network throughput and show that it provides better network throughput than the classical minimum power-based shortest path routing. We also develop an effective heuristic method to determine the minimum number of channels required for interference-free channel assignment.
\end{abstract}

Keywords: Channel assignment; Fairness; Interference-free; Maximum throughput; Multi-radio multi-channel; Wireless mesh networks

\section{Introduction}

In the classical approach to channel assignment in multi-radio multi-channel (MRMC) wireless mesh networks (WMNs) [1-7], the number of available nonoverlapping frequency channels is assumed to be fixed. In such schemes, two links that are within the interference range of each other could be assigned the same frequency, causing co-channel interference which degrades the network throughput.

We study the channel assignment problem in MRMC WMNs from a different perspective. First, we ensure interference-free communication among the mesh nodes by ensuring that links within the interference range of each other are assigned different channels. Second, instead of working with a fixed number of orthogonal frequency channels, we search for the minimum number of channels that provides an interference-free channel assignment which allows the mesh network to achieve the maximum throughput.

\footnotetext{
* Correspondence: auhchaud@sce.carleton.ca

Department of Systems and Computer Engineering, Carleton University, Ottawa, ON K1S 5B6, Canada
}

We assume a single mesh gateway (GW). All mesh nodes, except the GW, are sources of flow. The GW is the sink for all flows. We formulate the MRMC WMN routing problem as a mixed integer linear program (MILP) whose objective is to maximize the network throughput while maintaining fairness among the multiple flows subject to flow conservation, halfduplex, and node-degree constraints. The problem of assigning channels to the links involved in routing so that communication among mesh nodes is interference free is similar to the minimum coloring problem for the conflict graph, which is known to be NP-hard for general graphs [8]. This amounts to finding the smallest number of maximal independent sets (MaISs), where the number of channels required is equal to the number of MaISs. To minimize the number of channels required, we use our existing Select $x$ for less than $x$ topology control algorithm (TCA) [2] to build the connectivity graph (CG). By controlling network connectivity, it lowers the transmitted power as much as possible without sacrificing network throughput and so tends to reduce transmitted power throughout the network, which supports green networking for WMNs.

\section{实}


Specifically, the contributions of our work are as follows.

- We develop a method to determine the minimum number of non-overlapping frequency channels required for interference-free channel assignment given the locations of the mesh nodes and the number of their half-duplex radio interfaces. This is also the number of channels required to achieve the maximum network throughput ${ }^{\mathrm{a}}$.

- We show that our TCA-based approach for building the CG outperforms the classical maximum powerbased CG approach for all node-degree constraints in terms of the number of channels required as well as the links-to-channels ratio.

- We show that the multi-path routing approach significantly outperforms the minimum power-based shortest path (MPSP) routing approach in terms of network throughput at higher node-degree constraints.

- We develop and compare two effective new heuristics for interference-free channel assignment (CA).

The rest of the paper is organized as follows. Section 2 presents related work. Our model for the network architecture is given in Section 3. Section 4 explains the creation of the connectivity graph using the two different approaches. The problem formulation for multi-path routing and MPSP routing are also presented. The creation of the conflict graph using the protocolbased interference model is given in Section 5, which also presents two heuristic approaches for the solution of the channel assignment problem. Performance evaluation with results is given in Section 6. Conclusions and some directions for future work are given in Section 7.

\section{Related work}

There is a great deal of literature on improving the performance of MRMC WMNs by maximizing the network throughput [1-7]. In all of these studies, the number of available non-overlapping frequency channels is assumed to be fixed, so links within the interference range of each other could be assigned the same channel, causing co-channel interference that degrades network throughput. We provide interference-free communication among the mesh nodes by ensuring that links that fall within the interference range of each other are assigned different non-overlapping frequency channels. We then determine the minimum number of channels required to realize such interference-free channel assignment in order to achieve the maximum network throughput.
In addition to channel assignment, the schemes in [1-7] also deal with routing in MRMC WMNs. In the previous work $[1,2]$, we employed minimum powerbased minimum spanning trees and minimum powerbased shortest path trees for degree constrained routing. In [3], traffic is routed using either minimum-hop path routing or randomized multi-path routing. In [4], routing heuristics incorporate the impact of interface switching cost and a possible implementation using the dynamic source routing protocol is discussed. The optimized link state routing protocol is used in [5] for route selection. The scheme in [6] uses a flow rate computation method for routing, which aims to maximize the network throughput. For the performance evaluation of the channel assignment scheme in [7], the routes are computed statically using the smallest number of hops. In this paper, we formulate the routing problem as a MILP with the objective of maximizing the network throughput under fairness, flow conservation, halfduplex, and node-degree constraints.

The schemes in [2-7] focus on improving the throughput in MRMC WMNs without considering fairness. In [1], we proposed channel assignment algorithms to improve throughput as well as fairness. The scheme in [9] achieves a good trade-off between throughput and fairness even though it does not find an absolutely even distribution. The authors have shown in [10] that the network throughput as well as fairness increases as the number of available radio interfaces per router or the number of available orthogonal frequency channels increases. The approach in [11] deals with congestion control and channel assignment and achieves significant gains in terms of network utilization and establishing fairness. In [12], the authors deal with the problem of joint channel assignment, link scheduling, and routing for throughput optimization, and show that the fairness and throughput achieved by their method is within a constant factor of the optimum value.

An algorithm is proposed in [13] for joint channel, capacity, and flow assignment in MRMC WMNs. It first tries to maximize the fairness and then uses the remaining unused network resources to maximize the overall network throughput. The divide-and-conquer approach in [14] splits the joint routing and channel assignment problem into separate sub-problems. This significantly improves fairness among the traffic flows. Throughput and fairness do not normally go hand in hand, and increasing one generally decreases the other. Our proposed approach, however, achieves maximum network throughput for MRMC WMNs and at the same time ensures fairness among the network flows.

Algorithms for solving the maximum independent set (MIS) problem have been widely used for resource allocation in multi-hop wireless networks. The scheduling 
scheme in [15] uses independent sets for feasible link scheduling in TDMA-based WMNs. The method in [9] uses maximal independent sets for link scheduling in multi-channel WMNs. In [16], the authors propose a polynomial time approximation scheme for computing an independent set from the link interference graph, as large as $(1-\varepsilon)$ times the cardinality of the MIS. Given the number of channels among other inputs, the method is then used to develop a channel assignment for MRMC WMNs such that the number of links in the communications graph that can be active simultaneously is maximized. In [17], maximal weighted independent set solutions are used to develop an algorithm for link scheduling in multi-radio multi-channel multi-hop wireless networks. A polynomial computing method in [18] searches for the critical maximal independent set that needs to be scheduled for optimal resource allocation. In [19], the authors use solutions for the maximum weighted independent set problem to develop approximation algorithms for link scheduling, and compute a maximum (concurrent) multi-flow in multi-radio multi-channel multi-hop wireless networks. We solve the minimum coloring problem heuristically by repeatedly solving the MaIS sub-problem to determine the minimum number of channels required for interference-free CA in MRMC WMNs.

\section{Network architecture}

We assume that each mesh node is equipped with multiple radio interfaces. One of these radios is used for control traffic, while the others are used for data traffic. We define the node-degree of a mesh node as the number of neighbors with which it can communicate data traffic simultaneously. For example, a node-degree of 2 means that each mesh node is equipped with two radio interfaces for data traffic and can communicate with at most two of its neighbors simultaneously.

The radio interfaces are assumed to be half-duplex; hence, a mesh node cannot send and receive at the same time using the same radio interface. It is assumed that each radio interface of a multi-radio mesh node is equipped with an omni-directional antenna and that the radio interfaces of nodes can be tuned to different nonoverlapping frequency channels. The control radios of all nodes are tuned to a common frequency channel for communication of the control traffic.

\section{Routing problem}

\subsection{Connectivity graph}

We compare two different approaches for building the CG $C(V, E)$, where vertices $V$ correspond to the wireless nodes and the edges $E$ correspond to the wireless links between the nodes. The first approach is the classical way based on maximum transmission power, while the second approach uses our Select $x$ for less than $x$ TCA.
The Select $x$ for less than $x$ TCA builds a CG using topology control to mitigate the co-channel interference and enhance spatial channel reuse while preserving network connectivity. Each mesh router (MR) broadcasts a Hello message containing its node ID and position over the control channel using the control radio at maximum power. From the information in the received Hello messages, each MR arranges its neighboring nodes in ascending order of their distance. The result is the maximum power neighbor table (MPNT). Then, each MR sends its MPNT along with its position and node ID to the GW over the control channel. For each MR in the network, the GW builds a direct neighbor table (DNT) by selecting at least $x$ nearest nodes for that MR. If required, the GW then converts some uni-directional links in the DNT of a mesh node into bi-directional links, which results in the final neighbor table (FNT) [2]. Bi-directional links are required for link-level acknowledgments and to ensure the existence of reverse paths.

We build the CG for the maximum power (MP) based approach using the MPNT and the CG for the TCAbased approach using the FNT. Node locations are assumed to be known. In order to achieve a strongly connected topology, we assume a maximum transmission range of $164 \mathrm{~m}$ for all mesh nodes.

\subsection{Problem formulation for multi-path routing}

We formulate the multi-path routing problem in multiradio WMNs as a MILP. We call the index $p \in P$ a commodity. Let

- $P$ be the commodities, i.e., source-destination pairs $\left(s_{1}, t_{1}\right), \ldots . .,\left(s_{B} t_{P}\right) ;$

- $f_{i j}^{p}$ be a variable denoting the amount of flow of commodity $p$ on link $l_{i j}$;

- $f_{s}{ }^{p}$ be a variable denoting the amount of inflow of commodity $p$ from the source of $p$;

- $f_{d}^{p}$ be a variable denoting the amount of outflow of commodity $p$ from the sink of $p$;

- $c_{i j}$ be an input parameter denoting the capacity of link $l_{i j}$ where $l_{i j} \in E$;

- $z_{i j}$ be a binary variable such that $z_{i j} \in\{0,1\}$ is 1 when the link $l_{i j}$ is used for routing and 0 otherwise;

- $d c$ be an input parameter denoting the constraint on the node-degree of the mesh routers, such that $d c \in$ $\{2,3,4,5,6\}$;

- $\operatorname{cost}_{i j}$ be an input parameter containing a cost of 0.0001 for each link $l_{i j}$;

- demand $_{s d}$ be an input parameter representing flow demands between the source-sink pairs and is equal to 1 for all commodities; and

- $y$ be a variable denoting the multiplier on the unit flow demand of the commodities. 


\subsubsection{Objective}

Given the connectivity graph and unit flow demand between the source-sink pairs, the objective is to maximize $y$, which is the multiplier on the unit flow demand of the commodities, so as to achieve the maximum total flow in the network using multiple paths between a sourcesink pair:

$$
\max \left(y-\sum_{l_{i j}}\left(z_{i j} \times \operatorname{cost} 1_{i j}\right)\right)
$$

The small value of $\operatorname{cost}_{i j}$ in (1) prevents redundant flow-loops and does not affect the result.

\subsubsection{Constraints}

The following are the flow balance constraints for the source nodes, sink (GW) node, and the intermediate nodes, respectively:

$$
\sum_{j} f_{s j}^{p}-\sum_{i} f_{i s}^{p}-f_{s}^{p}=0 \quad \text { for all } s \text { and for all } p
$$

where $s, i, j \in V$;

$$
f_{d}^{p}+\sum_{i} f_{i d}^{p}-\sum_{j} f_{d j}^{p}=0 \quad \text { for all } d \text { and for all } p
$$

where $d, i, j \in V$;

$$
\sum_{j} f_{j i}^{p}-\sum_{k} f_{i k}^{p}=0 \quad \text { for all } i \text { and for all } p
$$

where $i \in V \backslash\left\{s_{p}, d_{p}\right\}$ and $p \in P$.

The following represent the constraints on the nodedegree of the mesh routers in the WMN. At any mesh node in the network, there is a limit on the number of radio interfaces and hence on the number of incoming plus outgoing links. If the limit is 4 , i.e., if $d c=4$, then at each mesh node $n_{i}$ :

$$
\sum_{k} z_{k i}+\sum_{j} z_{i j} \leq 4 \quad \text { for all } i
$$

where $i \in V$.

Varying $x$ in the Select $x$ for less than $x$ TCA for different node-degree constraints ensures that the total amount of flow in the network increases equally for the TCA-based and MP-based CG approaches with an increase in the node-degree constraint. It creates a certain amount of connectivity in the CG for a certain nodedegree constraint and hence a certain number of links for the GW. For example, for node-degree constraints of 2 and 3, we use the Select 3 for less than 3 TCA to ensure at least three links for the GW in the CG; for the node-degree constraint of 4 , we use the Select 4 for less than 4 TCA to ensure at least four links for the GW in the CG, and so on. Since the Select 2 for less than 2 TCA mostly leads to a disconnected network in the case of random and controlled random topologies, we use the Select 3 for less than 3 TCA for the node-degree constraint of 2 . The amount of total flow in the network depends on the number of links for the GW (sink) node for a given node-degree constraint. For example, if the capacity of each link is 24 , then the maximum possible total network flow, i.e., the maximum network throughput, is 48 for a node-degree constraint of 2 .

The following constraints introduce fairness among the flows of multiple commodities and ensure that they are maximized equally:

$$
f_{s}^{p} \geq y \times \operatorname{demand}_{s d} \quad \text { for all } s \text { and } d \text { and for all } p
$$

where $s \in\{$ sources $\}, d \in\{$ sinks $\}$, and $p \in P$.

The following constraints represent the half-duplex nature of the radio interfaces of the mesh routers, which means that a link can be used for either transmission or reception but not for both:

$$
z_{i j}+z_{j i} \leq 1 \quad \text { for all } i \text { and } j
$$

The link capacity constraints ensure that the sum of flows of commodities on a link does not exceed the link capacity:

$$
\sum_{p} f_{i j}^{p} \leq c_{i j} \times z_{i j} \quad \text { for all } i \text { and } j \text { and for all } p
$$

All link capacities are assumed to be 24 based on the fact that the maximum goodput of an IEEE 802.11a link operating at a maximum data rate of $54 \mathrm{Mbps}$ is approximately $24 \mathrm{Mbps}$.

$$
c_{i j} \geq 0 \quad \text { for all } i \text { and } j
$$

The flow of a commodity $p$ on a link is non-negative but is not required to be an integer.

$$
f_{i j}^{p} \geq 0 \text { for all } i \text { and } j \text { and for all } p
$$

We use the AMPL language [20] to model the multipath routing problem and IBM CPLEX 12.2 [21] to solve the resulting problem. 


\subsection{Problem formulation for MPSP routing}

We formulate the MPSP routing problem in two stages. In the first stage, shortest paths are determined between the source-sink pairs. The metric for path selection is minimum power. For the two CG approaches, the gateway calculates the minimum power required to reach each of the nodes in the FNT or the MPNT of a node by using the appropriate propagation model. The free space model is used for short distances, and the two-ray ground reflection model is used for longer distances, depending on the value of the Euclidean distance in relation to the cross-over distance [22]. If the distance between two nodes $u$ and $v$ is less than or equal to the cross-over distance, i.e., $d(u, v) \leq$ cross_over_dist, the free space model is used, whereas if $d(u, v)>$ cross_over_dist, the two-ray model is used. In the second stage, the flows of individual commodities are maximized equally so as to achieve the maximum total flow in the network, using the shortest paths determined in the first stage.

\subsubsection{First stage}

We formulate this stage as the following MILP. Let

- $\operatorname{cost} 2_{i j}$ be an input parameter containing the cost of each link $l_{i j}$ which is minimum power;

- supply be an input parameter which is 1 for sources; and

- demand $_{i}$ be an input parameter which is -1 for the sink (gateway).

4.3.1.1. Objective Shortest paths are determined between the source-sink pairs using the following objective:

$$
\min \sum_{p}\left(\sum_{l_{i j}}\left(f_{i j}^{p} \times \operatorname{cost} 2_{i j}\right)\right)
$$

4.3.1.2 Constraints The following are the flow balance constraints for this stage:

$$
\sum_{j} f_{i j}^{p}=\operatorname{supply}_{i}+\sum_{h} f_{h i}^{p} \quad \text { for all } i \text { and for all } p
$$

where $i \in V$ is the source of $p$;

$$
\sum_{j} f_{i j}^{p}=\operatorname{demand}_{i}+\sum_{h} f_{h i}^{p} \quad \text { for all } i \text { and for all } p
$$

where $i \in V$ is the sink of $p$;

$$
\sum_{j} f_{i j}^{p}=\sum_{h} f_{h i}^{p} \quad \text { for all } i \text { and for all } p
$$

where $i \in V \backslash\left\{s_{p}, d_{p}\right\}$.
The node-degree, half-duplex, and capacity constraints are exactly the same as in (5), (7), and (8), respectively. Note that because of the integer demand and supply flows and the unimodularity property of the network matrix, the continuous flows $f_{i j}^{p}$ will have binary values in the solution.

\subsubsection{Second stage}

We formulate this stage as the following linear program:

4.3.2.1 Objective The multiplier on the unit flow demand of the individual commodities is maximized to achieve the maximum total flow in the network, using the shortest paths determined in the first stage:

$$
\max y
$$

The additional term in (1) is not required in (15) since there is no possibility of flow-loops due to pre-established shortest paths.

4.3.2.2. Constraints The constraints for this stage are exactly the same as in (2), (3), (4), and (6). In addition, this stage requires the following capacity constraint:

$$
\sum_{p} f_{i j}^{p} \leq c_{i j} \quad \text { for all } i \text { and } j \text { and for all } p
$$

As in the case of multi-path routing, we use the AMPL language to model the MPSP routing problem and IBM CPLEX 12.2 to solve the resulting problem.

\section{Channel assignment problem 5.1. Conflict graph}

We use the protocol-based interference model [23] to build the conflict graph, which is widely used for modeling interference in wireless networks $[11,12,18,19]$. The input to the conflict graph consists of the links involved in routing, i.e., the output of the routing problem, and the node locations.

Let $d_{i j}$ denote the distance between nodes $n_{i}$ and $n_{j}$, $R_{i}$ be the transmission range of node $n_{i}$, and $R_{i}^{\prime}$ be the interference range of node $n_{i}$. In the conflict graph $F$, the vertices correspond to the links in the connectivity graph $C$. An edge between the vertices $l_{i j}$ and $l_{p q}$ in $F$ indicates that the links $l_{i j}$ and $l_{p q}$ in $C$ cannot be active simultaneously. Note that links $l_{i j}$ and $l_{p q}$, which are involved in routing, are bi-directional links at the MAC and physical layer levels. So, a link $l_{i j}$ which is involved in routing is checked eight times with every other link that is involved in routing while building the conflict graph. An edge is 
drawn between the vertices $l_{i j}$ and $l_{p q}$ if any of the following is true:

- $d_{i p} \leq R_{i}^{\prime}$ or $d_{i q} \leq R_{i}^{\prime}$ or $d_{j p} \leq R_{j}^{\prime}$ or $d_{j q} \leq R_{j}^{\prime}$ or

- $d_{p i} \leq R_{p}^{\prime}$ or $d_{p j} \leq R_{p}^{\prime}$ or $d_{q i} \leq R_{q}^{\prime}$ or $d_{q j} \leq R_{q}^{\prime}$.

While building the conflict graph, we assume that the interference range is twice the transmission range.

\subsection{Maximum independent set problem and the minimum coloring problem}

An independent set of a graph $G$ is a subset of vertices of $G$ such that none of the vertices in the subset share an edge. In the conflict graph $F$, an independent set of vertices indicates a set of links in $C$ which can be active simultaneously. An independent set is said to be $\max$ imal if it is not a subset of any larger independent set or maximum if there is no larger independent set in the graph (i.e., it is an independent set with maximum cardinality). The MIS problem consists of finding the largest subset of vertices of a graph such that none of these vertices are connected by an edge.

A coloring of the conflict graph $F$ is an assignment of colors (channels) to vertices such that adjacent vertices receive different colors. The minimum coloring problem is the problem of computing a coloring of the vertices in the conflict graph $F$ using as few distinct colors as possible; this is the same as the problem of finding the minimum number of channels to use such that there is no interference. The minimum coloring problem is well known to be NP-hard for general graphs [8]. A greedy heuristic for its solution consists of these steps: (1) find a maximal independent set of vertices and assign the members of this set to the same channel, (2) remove these vertices from the conflict graph, and (3) repeat until all vertices are colored (assigned a channel). The number of channels required to achieve interferencefree communication among the mesh nodes is equal to the number of MaISs.

Since finding a maximum independent set is itself NPhard [24], we also use heuristic algorithms for this step of the solution to the interference-free channel assignment problem.

\subsection{MalS-based heuristics for CA}

We use three greedy heuristic algorithms to find MaISs. Algorithm 1 Maximum node-degree start selects a vertex from the conflict graph with the maximum node-degree and introduces that vertex into the maximal independent set under construction. The algorithm then checks the other vertices of the conflict graph and puts them in the set if they do not have an edge with the vertices already in the set. The worst-case computational complexity of Algorithm 1 to find a MaIS is $O\left(m^{2}\right)$ where $m$ is the number of nodes in the conflict graph.

\section{Algorithm 1 Maximum node-degree start}

\section{Inputs:}

- $\quad L$ : set of links involved in routing

- $F\left(V_{F}, E_{F}\right)$ : conflict graph

\section{Outputs:}

- $V_{\text {MaIs: }}$ MaIS of vertices

- $L_{\text {Mals}}$ : Set of links in $C$ corresponding to vertices in MaIS

- $\quad c$ : number of MaISs

\section{BEGIN}

$c \leftarrow 0$

$m \leftarrow|L|=\left|V_{F}\right|$

While $m \geq 1$ :

If $F$ is fully connected or $m=1$ :

For $d=1$ to $m$ :

Initialize an empty $V_{\text {MaIS }}$ and $L_{\text {MaIS }}$ Add vertex $d$ to $V_{\text {MaIS }}$ and the corresponding link to $L_{\text {MaIS }}$ Output $V_{\text {MaIS }}$ and $L_{\text {MaIS }}$ $c \leftarrow c+1$

end For

end If

Output $c$ and exit

Initialize an empty $V_{\text {MaIS }}$ and $L_{\text {MaIS }}$

Add vertex of $F$ with the max. node-degree to $V_{\text {MaIS }}$ and the corresponding link to $L_{\text {MaIS }}$

For $i=1$ to $m$ :

If vertex $i$ does not have an edge with vertices in $V_{\text {MaIS }}$ : Add vertex $i$ to $V_{\text {MaIS }}$ and the end If corresponding link to $L_{\text {MaIS }}$

\section{end For}

Output $V_{\text {MaIS }}$ and $L_{\text {MaIS }}$

$c \leftarrow c+1$

$V_{F} \leftarrow V_{F} \backslash V_{\text {Mals }}$

$L \leftarrow L \backslash L_{\text {MaIS }}$

$m \leftarrow|L|$

end While

Output $c$

END

Algorithm 1 Minimum node-degree start starts by selecting a vertex from the conflict graph with the minimum node-degree. Note that if the conflict graph has multiple vertices with the maximum node-degree (Algorithm 1 Maximum node-degree start) or with the minimum node-degree (Algorithm 1 Minimum node-degree start), then the starting vertex is selected randomly from among them. After removing a MaIS from the conflict graph, ties for the starting vertex in the revised conflict graph are again broken randomly if required. Algorithm 1 
Random start starts by selecting a vertex at random from the conflict graph.

These randomized heuristics find MaISs very quickly but may return different results each time, so we run them multiple times. For a given topology of MRs, we run each of the three algorithm variants 25 times on the conflict graph and take the best solution over all 75 runs.

\subsection{MIS-based heuristics for CA}

The greedy minimum coloring heuristic presumably works best if the maximum independent set can be found at each step instead of just a maximal independent set. We tested Wilf's algorithm [25] for finding the maximum independent set of a given graph $G$. It starts by choosing a vertex $v^{*}$ from the graph which has the highest nodedegree. If $v^{*}$ has at least two neighbors, the computational complexity of Wilf's algorithm to find a MIS in the given graph is $O\left(1.47^{m}\right)$, which reduces to $O\left(1.39^{m}\right)$ if $v^{*}$ has at least three neighbors, where $m$ is the number of nodes in the graph. After selecting $v^{*}$, the algorithm builds two sets. The set $n 1$ contains all the nodes of the graph except $v^{*}$ and the set $n 2$ excludes both $v^{*}$ and $N\left(v^{*}\right)$, the neighborhood of $v^{*}$, i.e., the nodes that are connected to $v^{*}$ by an edge. maxset is the set which has the higher cardinality of the two sets. The vertices in maxset (and their incident edges) are deleted from $G$, and the procedure is repeated until $G$ has no edges. The MIS consists of the vertices in the disconnected $G$. The pseudo-code of our procedure using Wilf's algorithm is shown in Algorithm 2.

\section{Algorithm 2 Procedure of finding MISs using Wilf's algorithm}

\section{Inputs:}

- $\quad L$ : set of links involved in routing

- $F\left(V_{F}, E_{F}\right)$ : conflict graph

\section{Outputs:}

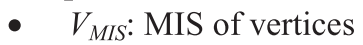

- $L_{M I S}$ : Set of links in $C$ corresponding to vertices in MIS

- $c$ : number of MISs

\section{BEGIN}

$c \leftarrow 0$

$m \leftarrow|L|=\left|V_{F}\right|$

While $m \geq 1$ :

If $F$ is fully connected or $m=1$ :

For $d=1$ to $m$ :

Initialize an empty $V_{M I S}$ and $L_{M I S}$

Add vertex $d$ to $V_{M I S}$ and the

corresponding link to $L_{M I S}$

Output $V_{M I S}$ and $L_{M I S}$

end For

$$
c \leftarrow c+1
$$

Output $c$ and exit

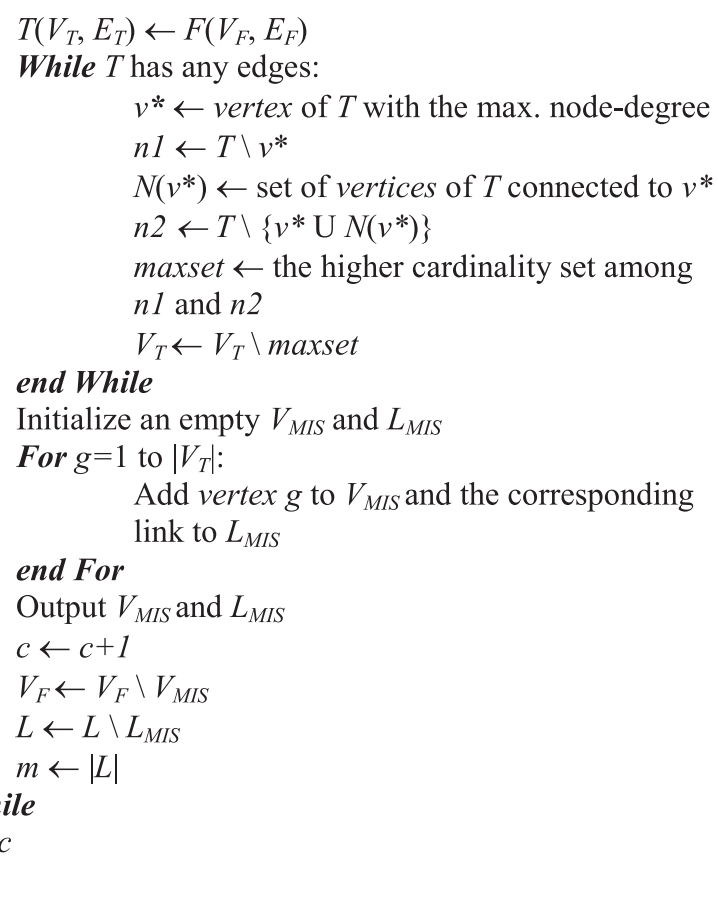

Note that the procedures related to routing and channel assignment are carried out at the GW, which then sends each MR its channel assignment and routing information over the control channel using the control radio. Based on the channel assigned to an MR to communicate with a neighbor and its distance to that neighbor, each MR applies power control and adjusts its transmission power accordingly.

\section{Performance evaluation}

We evaluate the performance of the TCA-based vs. MPbased CG approach, multi-path vs. MPSP routing, and the MaIS-based vs. MIS-based heuristics for CA, based on network throughput, fairness, solution time of the routing stage in clock seconds, number of channels required (NCR), and links-to-channels ratio (LCR), i.e., the ratio of the number of links involved in routing to the number of channels required. Note that an average LCR of 2 for a topology means that on the average, two links are assigned the same channel for that topology, so a higher LCR indicates better performance.

\subsection{Network topology}

A controlled random topology (CRT) is used for the evaluation. A $500 \mathrm{~m} \times 500 \mathrm{~m}$ physical terrain is divided into cells, and an MR is placed randomly within each cell using a uniform random distribution. Twenty-five different CRTs consisting of 36-node networks are considered. Irrespective of its location, node 15 is the GW for all CRTs. All mesh nodes, except the GW, are sources of flow. As stated earlier, the GW is the sink for all flows. 


\subsection{Experimental results}

\subsubsection{TCA-based vs. MP-based CG approach}

We compare the performances of the TCA-based vs. MPbased CG approaches for different node-degree constraints. For both CG approaches, we use multi-path routing in combination with the MaIS-based heuristic for channel assignment. All CPLEX solver parameters are at their default settings, except mipgap $=0.01$. This speeds the MILP solution at the possible expense of a small degradation in the objective value, though we found the optimum in all cases. For the TCA-based CG approach, if the solver finds a sub-optimum solution for the multi-path routing problem for node-degree constraints of 2 or 3, we move to a higher TCA to build the CG, i.e., Select 4 for less than 4 TCA or Select 5 for less than 5 TCA, until an optimum solution is found. Note that for a node-degree constraint of 2 , the maximum value of total network flow (maximum network throughput) is 48; for a node-degree constraint of 3 , it is 72 ; and so on.

We collected mean values and statistics on the $95 \%$ confidence intervals for all measures. If the $95 \%$ confidence interval is tightly grouped around the mean, then it is not reported in a table. Most measures are graphed so that trends are immediately apparent.

6.2.1.1. Fairness The amount of flow of each commodity reaching the GW (sink) is equal to $y \times \operatorname{demand}_{s d}$ as per (6). However, due to a unit flow demand between each source-sink pair, the value of $y$ in Table 1 represents the amount of flow from each source reaching the GW. This indicates that all of the 35 flows in the network are maximized equally. Jain's measure of fairness among the network flows [26] $F_{J}$ is given by:

$$
F_{J}=\left(\sum_{i=1}^{N} x_{i}\right)^{2} /\left(N \times \sum_{i=1}^{N} x_{i}^{2}\right)
$$

Table 1 Total flow (TCA-based CG vs. MP-based CG approach)

\begin{tabular}{cccc}
\hline Node-degree constraint & CG approach & $\boldsymbol{y}$ & Total flow \\
\hline 2 & MP-based & 1.3714 & 48 \\
& TCA-based & & \\
3 & MP-based & 2.0571 & 72 \\
& TCA-based & & 96 \\
4 & MP-based & 2.7428 & 120 \\
5 & TCA-based & & 144 \\
6 & MP-based & 3.4285 & \\
& TCA-based & & \\
& MP-based & 4.1142 & \\
& TCA-based & & \\
&
\end{tabular}

where $x_{i}$ is the throughput of flow $i$ and $N$ is the total number of flows (sources) in the network. The flows are maximized equally for both CG approaches, so absolute fairness is achieved and the value of $F_{J}$ is 1 in all CRTs for all node-degree constraints.

6.2.1.2. Network throughput The results in Table 1 indicate that the total flow in the network (the flow reaching the gateway (sink)) increases equally for the MP-based and TCA-based CG approaches with an increase in the node-degree constraint. For a node-degree constraint of 2 in Table 1, $y$ is 1.3714. The total flow in the network, i.e., the network throughput, is equal to the number of sources times $y$, i.e., $35 \times 1.3714=48$, for both CG approaches, which is equal to the maximum total network flow (maximum network throughput) for a nodedegree constraint of 2 . In fact, the network throughput achieved is maximum for all node-degree constraints for both CG approaches, as shown in Table 1.

Our centralized approach leads to an optimum solution in terms of network throughput while also ensuring fairness among the network flows since the required global network information is available at the GW.

6.2.1.3. Solution time of routing stage Table 2 compares the solution time in clock seconds of the multi-path routing stage for the two CG approaches, showing the average solution times for the 25 CRTs along with the 95\% confidence interval $(\mathrm{CI})$ at different node-degree constraints. The TCA-based CG approach is clearly faster than the MP-based CG approach.

6.2.1.4. Number of channels required Figure 1 shows that the TCA-based CG approach requires fewer channels than the MP-based CG approach for all node-degree constraints. Using multi-path routing in combination with the MaIS-based CA heuristic at a node-degree constraint of 2,

Table 2 Solution time (TCA-based CG vs. MP-based CG approach)

\begin{tabular}{cccc}
\hline $\begin{array}{c}\text { Node-degree } \\
\text { constraint }\end{array}$ & CG approach & Solution time (s) & $\begin{array}{c}\mathbf{9 5 \%} \mathbf{C l} \text { for } \\
\text { solution time }\end{array}$ \\
\hline 2 & MP-based & 656.33 & $296.43-1,016.22$ \\
& TCA-based & 138.24 & $102.00-174.49$ \\
3 & MP-based & 11.31 & $10.32-12.30$ \\
& TCA-based & 3.04 & $2.62-3.45$ \\
4 & MP-based & 10.89 & $9.83-11.96$ \\
& TCA-based & 3.70 & $3.13-4.28$ \\
5 & MP-based & 9.74 & $8.32-11.17$ \\
& TCA-based & 3.61 & $2.80-4.43$ \\
& MP-based & 8.82 & $7.26-10.38$ \\
& TCA-based & 3.99 & $3.10-4.89$ \\
\hline
\end{tabular}




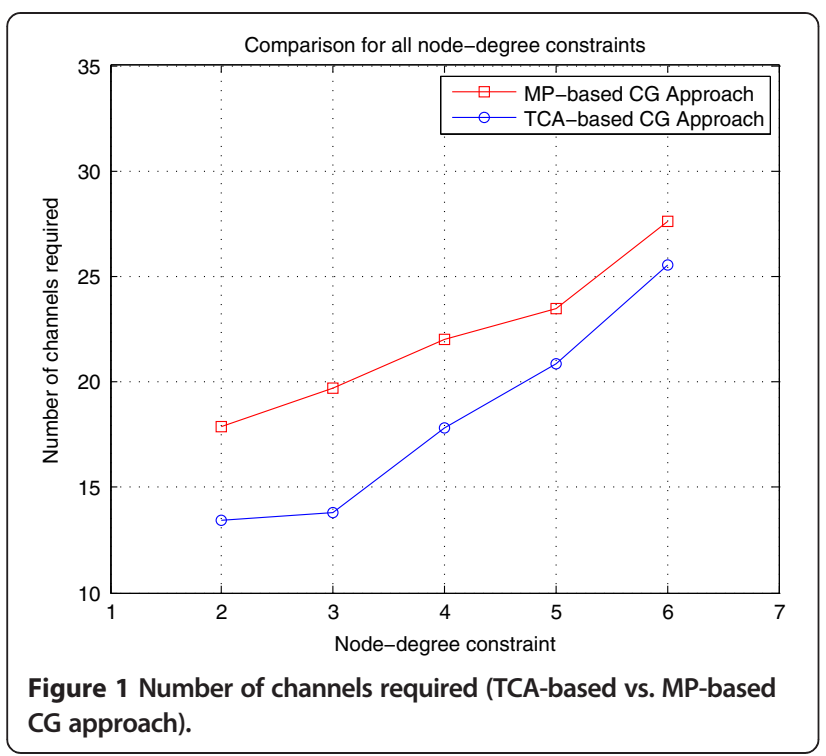

the TCA-based CG approach uses one third fewer channels than the MP-based CG approach.

6.2.1.5. Links-to-channels ratio Figure 2 shows that the LCR for the TCA-based CG approach is higher than that for the MP-based CG approach for all node-degree constraints. Using multi-path routing in combination with the MaIS-based CA heuristic at a node-degree constraint of 2, the LCR for the TCA-based CG approach is one third higher than that for the MP-based CG approach.

As the node-degree constraint increases so does the number of links emanating from a mesh node. Since the links emanating from a node must be assigned different channels, this increases the NCR as well as

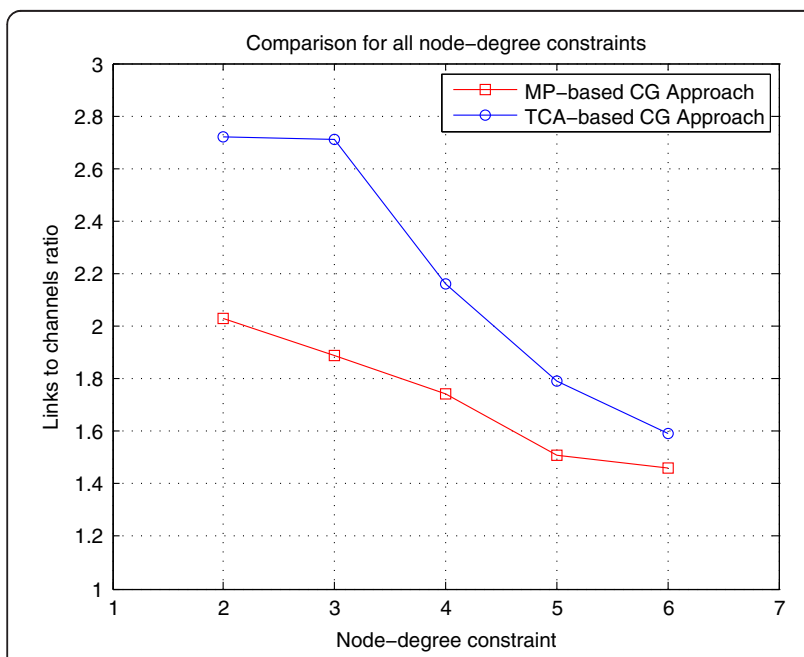

Figure 2 Links-to-channels ratio (TCA-based vs. MP-based CG approach). decreasing the LCR for both CG approaches, as shown in Figures 1 and 2. However, the TCA-based approach still outperforms the MP-based approach by controlling the network connectivity using topology control, as shown next.

The average node-degree (AND) for the entire network is calculated as:

$$
\mathrm{AND}=\frac{1}{N} \sum_{j=1}^{N} q_{j}
$$

where $q$ is the number of neighbors of a mesh node and $N$ is the total number of mesh nodes in the network. We define transmission range-average node-degree (TR-AND) and interference range-average node-degree (IR-AND) as the average number of transmission range neighbors and the average number of interference range neighbors of nodes, respectively. Figures 3 and 4 compare TR-AND (connectivity) and IR-AND (interference) for the two CG approaches, respectively. With the TCA-based approach, the connectivity and interference can be controlled, as can be seen in these figures. However, as the network connectivity increases by increasing $x$ in the Select $x$ for less than $x$ TCA so does the interference in the network. This increases the NCR as well as decreasing the LCR at higher node-degree constraints with the TCA-based approach.

Since the solver finds a sub-optimum solution of the multi-path routing problem for a node-degree constraint of 2 with the TCA-based approach for many of the CRTs, we move from Select 3 for less than 3 TCA to a higher TCA to build the CG until an optimum solution is found. This is the reason for the higher connectivity

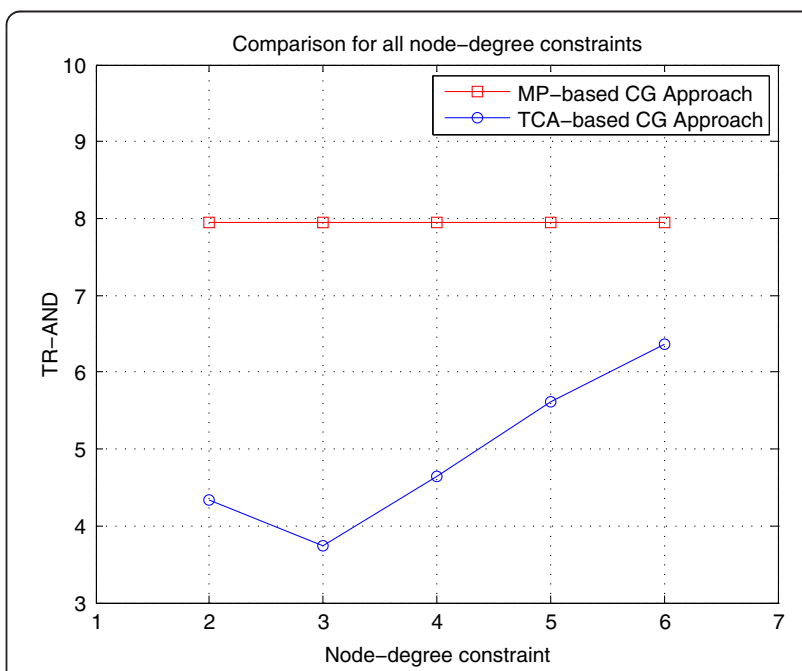

Figure 3 Transmission range-average node-degree (TCA-based vs. MP-based CG approach). 


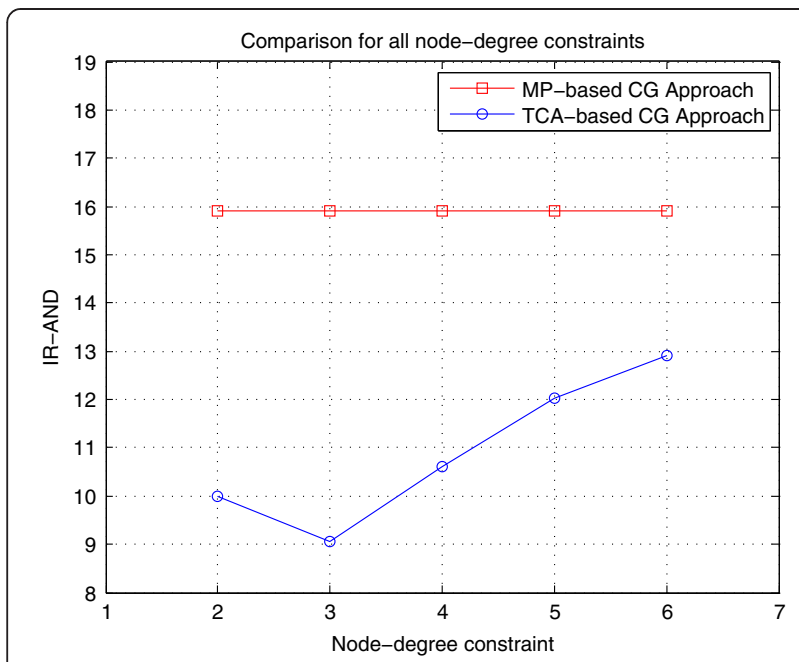

Figure 4 Interference range-average node-degree (TCA-based vs. MP-based CG approach).

and interference in Figures 3 and 4 for a node-degree constraint of 2 with the TCA-based approach.

\subsubsection{Multi-path vs. MPSP routing approach}

We compare the performances of the multi-path vs. MPSP routing approaches for different node-degree constraints. For each routing approach, we use the TCAbased CG as well as the MP-based CG in combination with the MaIS-based heuristic CA approach. For both routing approaches, we ask CPLEX to search for the first feasible solution, which significantly reduces the solution time at the cost of a small degradation in the network throughput, and set mipemphasis $=1$ in CPLEX. For the TCA-based CG approach, we use the Select 3 for less than 3 TCA for the node-degree constraint of 2 and 3, Select 4 for less than 4 TCA for the node-degree

Table 3 Total flow (multi-path routing vs. MPSP routing (usingTCA-based CG))

\begin{tabular}{ccccc}
\hline $\begin{array}{c}\text { Node-degree } \\
\text { constraint }\end{array}$ & Routing approach & $\boldsymbol{y}$ & Total flow & $\begin{array}{c}\mathbf{9 5 \%} \text { Cl for } \\
\text { total flow }\end{array}$ \\
\hline 2 & Multi-path & 1.160 & 40.59 & $37.95-43.23$ \\
3 & MPSP & 1.199 & 41.96 & $40.40-43.53$ \\
& Multi-path & 1.902 & 66.56 & $62.15-70.97$ \\
4 & MPSP & 1.314 & 46.00 & $42.19-49.82$ \\
& Multi-path & 2.382 & 83.37 & $78.19-88.55$ \\
5 & MPSP & 1.487 & 52.03 & $47.55-56.52$ \\
& Multi-path & 2.981 & 104.33 & $97.01-111.66$ \\
6 & MPSP & 1.603 & 56.11 & $51.33-60.89$ \\
& Multi-path & 3.593 & 125.74 & $116.67-134.81$ \\
& MPSP & 1.724 & 60.35 & $54.99-65.71$ \\
\hline
\end{tabular}

constraint of 4 , and so on. We are only interested in the first feasible solution for this comparison, so we do not move to a higher TCA in search of the optimum, unlike the previous comparison.

The MP-based CG performs poorly as compared to the TCA-based CG for both routing approaches in terms of solution time of the routing stage, NCR, and LCR and is slightly better than TCA-based CG in terms of network throughput. Therefore, for the sake of brevity, we present the results for each routing approach with TCA-based CG only in combination with the MaISbased CA heuristic.

6.2.2.1. Fairness As indicated by the results in Table 3, the $y$ values are maximized equally for all flows in both routing approaches. Absolute fairness is achieved for both routing approaches in all CRTs for all node-degree constraints.

6.2.2.2. Network throughput The results in Table 3 and Figure 5 indicate that the total amount of flow in the network increases for both routing methods with an increase in the node-degree constraint. However, the increase in network throughput is significantly higher with multi-path routing, especially at higher node-degree constraints. For example, at a node-degree constraint of 6 and using the TCA-based CG, the network throughput is $87.3 \%$ of the maximum network throughput of 144 using multi-path routing as compared to a network throughput of $41.9 \%$ of the maximum network throughput with MPSP routing. Note that the small degradation in the network throughput with multi-path routing is due to finding only the first feasible solution vs. finding

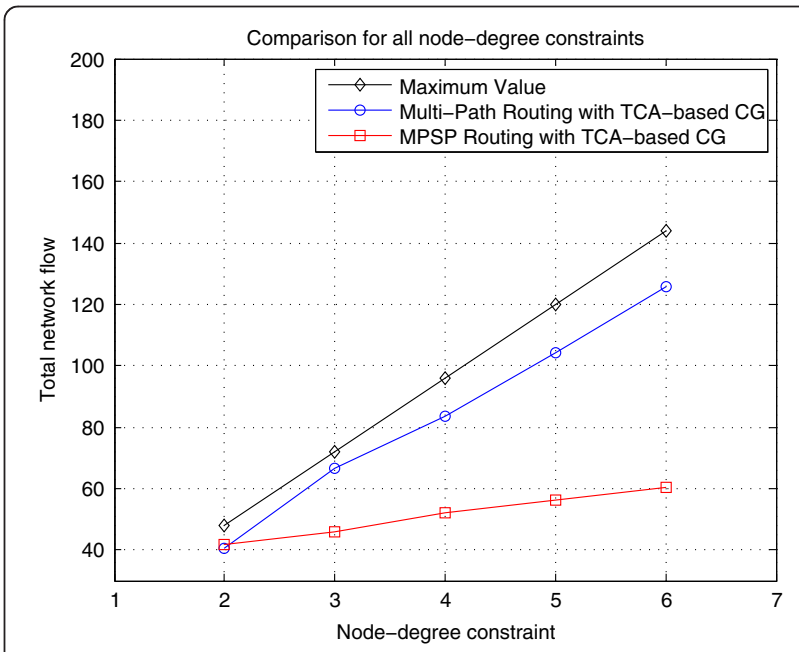

Figure 5 Total flow in the network (multi-path vs. MPSP routing approach (using TCA-based CG)). 
Table 4 Solution time (multi-path routing vs. MPSP routing (usingTCA-based CG))

\begin{tabular}{cccc}
\hline $\begin{array}{c}\text { Node-degree } \\
\text { constraint }\end{array}$ & Routing approach & Solution time (s) & $\begin{array}{c}\mathbf{9 5 \%} \text { Cl for } \\
\text { solution time }\end{array}$ \\
\hline 2 & Multi-path & 13.79 & $8.60-18.98$ \\
3 & MPSP & 1.64 & $1.47-1.82$ \\
& Multi-path & 1.68 & $1.48-1.88$ \\
4 & MPSP & 0.25 & $0.23-0.27$ \\
& Multi-path & 1.75 & $1.43-2.08$ \\
5 & MPSP & 0.24 & $0.24-0.25$ \\
& Multi-path & 1.89 & $1.37-2.42$ \\
6 & MPSP & 0.26 & $0.26-0.27$ \\
& Multi-path & 2.37 & $1.69-3.05$ \\
& MPSP & 0.28 & $0.28-0.29$ \\
\hline
\end{tabular}

the optimum solution. The lower network throughput of MPSP routing is mainly because maximizing the total network flow is not an objective while determining the shortest paths between the sources and the GW.

6.2.2.3. Solution time of routing stage Table 4 compares the two routing methods in terms of the solution time in clock seconds. For multi-path routing, the solution times are reduced as compared to those in Table 2, but there is a trade-off in the form of a decrease in the network throughput. As indicated in Table 4, MPSP routing solution times are smaller than those for multipath routing. Since the objective of the multi-path routing is to maximize the total network flow, it takes the solver more time to find the first feasible solution for it as compared to MPSP routing.

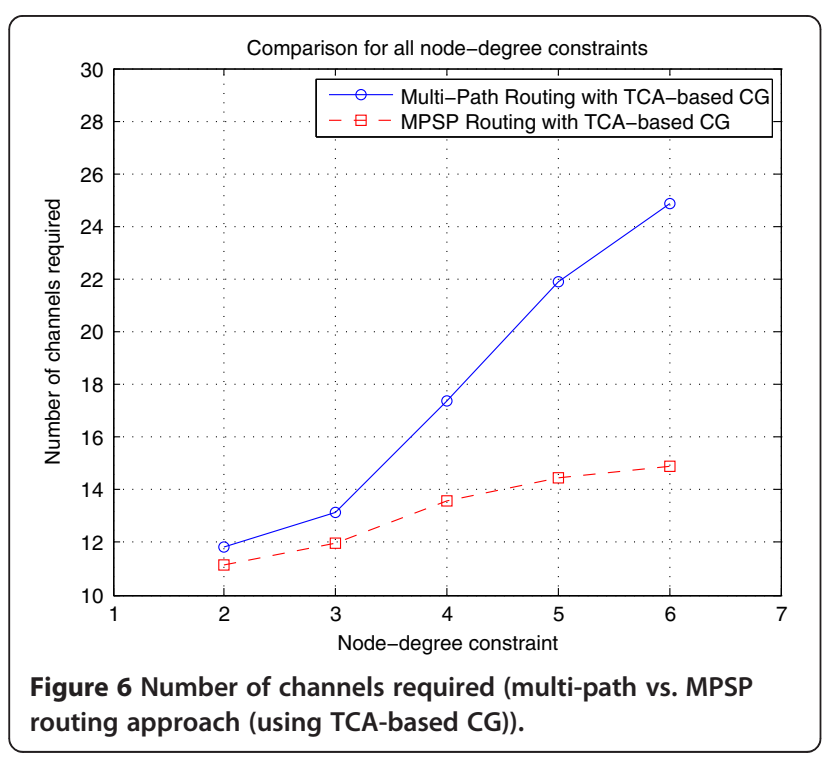

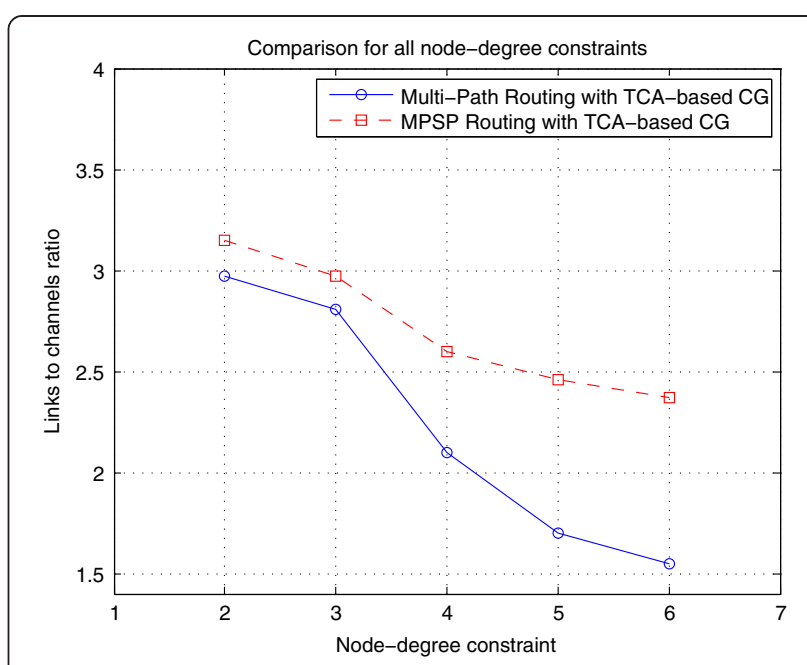

Figure 7 Links-to-channels ratio (multi-path vs. MPSP routing approach (using TCA-based CG)).

6.2.2.4. Number of channels required The results in Figure 6 indicate that fewer channels are required by MPSP routing than are required by multi-path routing.

6.2.2.5. Links-to-channels ratio Figure 7 shows that the LCR with MPSP routing is higher than that for multi-path routing.

Since the objective of multi-path routing is to maximize the total flow in the network by using multiple paths between the mesh nodes and the GW, it returns solutions in which more nodes have high node-degrees, which means more links (conflicts) in the conflict graph. This leads to a higher NCR and a lower LCR as compared to MPSP routing. Although MPSP routing has smaller solution times,

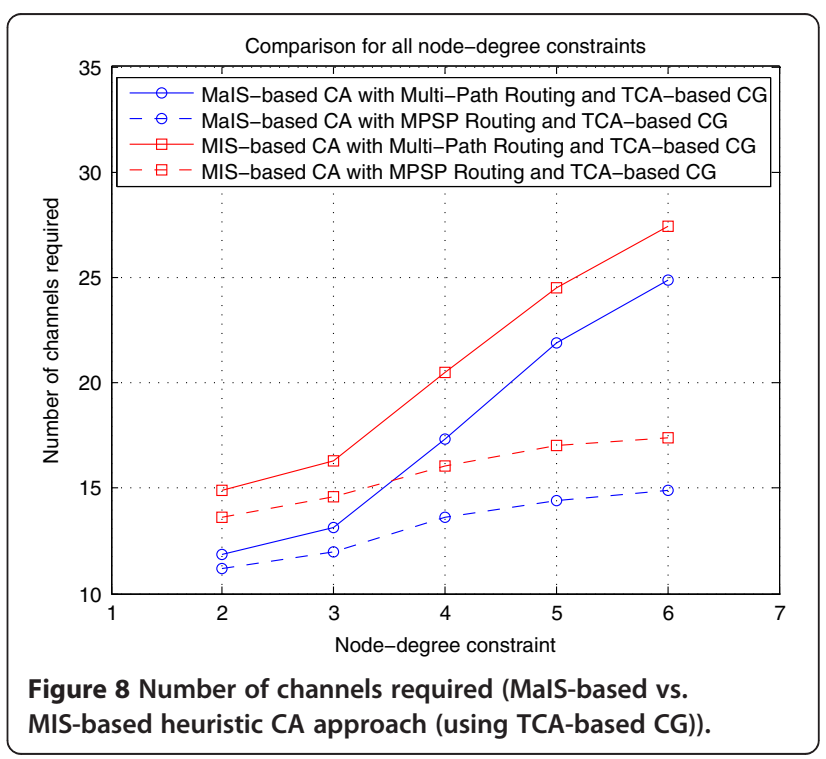


NCR, and LCR, it performs poorly vs. multi-path routing in terms of network throughput at higher node-degree constraints. There is a trade-off between the network throughput and the NCR for the two routing approaches. For example, using the TCA-based CG and a node-degree constraint of 6 , multi-path routing provides 2.08 times more network throughput than MPSP routing but is 1.67 times costlier than MPSP routing in terms of NCR.

\subsubsection{MalS-based vs. MIS-based heuristic approach for channel assignment}

We compare the performances of MaIS-based vs. MISbased heuristic approaches for CA for different nodedegree constraints based on NCR and LCR. For each CA approach, we use the two routing methods in combination with the two CG approaches. During routing, we search for the first feasible solution and use mipemphasis $=1$ in CPLEX to reduce the solution time.

Since MP-based CG mostly performs poorly as compared to the TCA-based CG for both CA methods, we present the results for each CA method with the TCAbased CG only in combination with the two routing approaches.

6.2.3.1. Number of channels required The results in Figure 8 show that fewer channels are required by the MaIS-based CA heuristic than the MIS-based CA heuristic. Using the TCA-based CG in combination with multi-path routing at a node-degree constraint of 2, the MaIS-based CA heuristic uses $25.6 \%$ fewer channels than the MIS-based CA heuristic.

6.2.3.2. Links-to-channels ratio The results in Figure 9 show that the LCR for the MaIS-based CA heuristic is

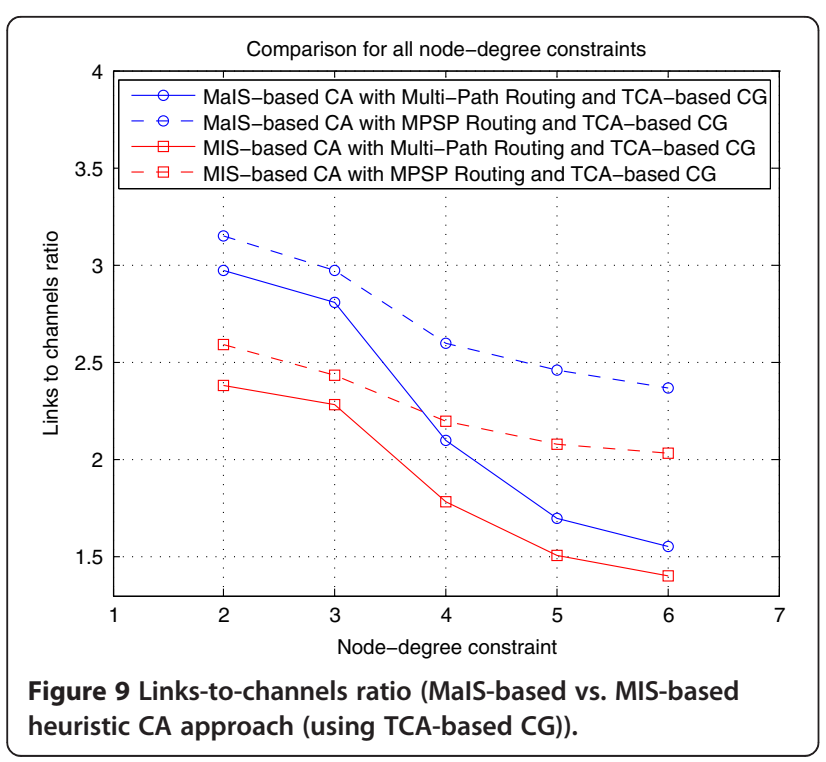

higher than that for the MIS-based CA heuristic. Using the TCA-based CG in combination with multi-path routing at a node-degree constraint of 2, the MaIS-based CA heuristic gives an LCR that is $24.7 \%$ higher than that for the MIS-based CA heuristic.

The MaIS-based heuristic not only outperforms the MIS-based heuristic in terms of NCR and LCR but is also much more efficient in terms of computational complexity. The MaIS-based heuristics return fewer channels than the MIS-based heuristics because the cardinalities of the MaISs are more even in size whereas the MIS-based heuristics first find a few large cardinality MISs, leaving a larger number of small MISs, for an overall larger number of channels.

\section{Conclusions}

We studied the problem of determining the minimum number of non-overlapping frequency channels required by the mesh network to achieve maximum network throughput while maintaining fairness. We used our existing Select $x$ for less than $x$ TCA to build the CG with the objective of enhancing the spatial channel reuse in order to minimize the number of channels required. We found that the TCA-based CG approach outperforms the classical approach of MP-based CG in terms of solution time of the routing stage, NCR, and LCR for all node-degree constraints. The TCA-based approach controls the network connectivity by controlling the neighborhoods of the mesh nodes, which leads to less overall transmitted power and better spatial channel reuse. We used multi-path routing to achieve the maximum network throughput. This significantly outperforms the classical approach of MPSP routing in terms of network throughput at higher node-degree constraints. However, we observed a trade-off between network throughput and NCR for the two routing approaches. With an increasing node-degree constraint, multi-path routing provides more network throughput than MPSP routing but becomes costlier than MPSP routing in terms of NCR at the same time. We used heuristic algorithms to determine the minimum number of channels required for interference-free $\mathrm{CA}$, with the MaIS-based heuristic CA outperforming the MIS-based heuristic CA in terms of NCR and LCR for all nodedegree constraints.

We plan to extend our work in future using the signal-to-interference ratio-based interference model for building the conflict graph. Further research will also focus on finding ways to significantly reduce the number of non-overlapping frequency channels required.

\section{Endnote}

${ }^{a}$ Preliminary work in this regard has been presented in ICCCN' 2013. 


\section{Competing interests}

The authors declare that they have no competing interests.

Received: 12 December 2012 Accepted: 2 August 2013

Published: 14 September 2013

\section{References}

1. AU Chaudhry, N Ahmad, RHM Hafez, Improving throughput and fairness by improved channel assignment using topology control based on power control for multi-radio multi-channel wireless mesh networks. EURASIP J. Wirel. Commun. Netw. (2012), 155 (2012)

2. AU Chaudhry, RHM Hafez, O Aboul-Magd, SA Mahmoud, Throughput improvement in multi-radio multi-channel 802.11a-based wireless mesh networks (IEEE Globecom 2010, Miami, 2010), pp. 1-5. doi: 10.1109/ GLOCOM.2010.5684193

3. A Raniwala, K Gopalan, T Chiueh, Centralized channel assignment and routing algorithms for multi-channel wireless mesh networks. ACM MC2R 8(2), 50-65 (2004)

4. P Kyasanur, N Vaidya, Routing and interface assignment in multi-channel multi-interface wireless networks (IEEE WCNC 2005, New Orleans, 2005), pp. 2051-2056. doi: 10.1109/WCNC.2005.1424834

5. K Ramachandran, E Belding, K Almeroth, M Buddhikot, Interference-aware channel assignment in multi-radio wireless mesh networks (IEEE INFOCOM 2006, Barcelona, 2006), pp. 1-12

6. S Avallone, IF Akyildiz, A channel assignment algorithm for multi-radio wireless mesh networks. Comput. Commun. 31(7), 1343-1353 (2008)

7. AP Subramanian, H Gupta, SR Das, C Jing, Minimum interference channel assignment in multiradio wireless mesh networks. IEEE Tran. Mob. Comput. 7(12), 1459-1473 (2008)

8. RM Karp, Reducibility among combinatorial problems. Complexity of computer computations (Plenum Press, New York, 1972), pp. 85-103

9. J Tang, G Xue, W Zhang, Cross-layer design for end-to-end throughput and fairness enhancement in multi-channel wireless mesh networks. IEEE Trans. Wireless Commun. 6(10), 3482-3486 (2007)

10. AHM Rad, WWS Wong, Cross-layer fair bandwidth sharing for multi-channel wireless mesh networks. IEEE Trans. Wireless Commun. 7(9), 3436-3445 (2008)

11. A Giannoulis, T Salonidis, E Knightly, Congestion control and channel assignment in multi-radio wireless mesh networks (IEEE SECON 2008, San Francisco, 2008), pp. 350-358. doi: 10.1109/SAHCN.2008.50

12. XY Li, A Nusairat, Y Wu, Y Qi, JZ Zhao, X Chu, Y Liu, Joint throughput optimization for wireless mesh networks. IEEE Trans. Mob. Comput. 8(7), 895-909 (2009)

13. V Ramamurthi, A Reaz, D Ghosal, S Dixit, B Mukherjee, Channel, capacity, and flow assignment in wireless mesh networks. Comput. Netw. $55,2241-2258(2011)$

14. V Gardellin, SK Das, L Lenzini, C Cicconetti, E Mingozzi, G Pamela, A divideand-conquer approach for joint channel assignment and routing in multiradio multi-channel wireless mesh networks. J. Parallel Distrib. Comput. 71, 381-396 (2011)

15. G Brar, D Blough, P Santi, Computationally efficient scheduling with the physical interference model for throughput improvement in wireless mesh networks (ACM Mobicom 2006, Los Angeles, 2006), pp. 2-13. doi: 10.1145/ 1161089.1161092

16. A Sen, S Murthy, S Ganguly, S Bhatnagar, An interference-aware channel assignment scheme for wireless mesh networks (IEEE ICC 2007, Glasgow, 2007), pp. 3471-3476. doi: 10.1109/ICC.2007.574

17. S Merlin, N Vaidya, M Zorzi, Resource allocation in multi-radio multi-channel multi-hop wireless networks (IEEE INFOCOM 2008, Phoenix, 2008), pp. 610-618. doi: 10.1109/INFOCOM.2008.110

18. H Li, Y Cheng, C Zhou, P Wan, Multi-dimensional conflict graph based computing for optimal capacity in mr-me wireless networks (IEEE ICDCS 2010, Genoa, 2010), pp. 774-783. doi: 10.1109/ICDCS.2010.58

19. P-J Wan, Y Cheng, Z Wang, F Yao, Multiflows in multi-channel multi-radio multihop wireless networks (IEEE INFOCOM 2011, Shanghai, 2011), pp. 846-854. doi: 10.1109/INFCOM.2011.5935308

20. R Fourer, DM Gay, BW Kernighan, AMPL: a modeling language for mathematical programming, 2nd edn. (Brooks/Cole, Belmont, CA, 2003)

21. IBM ILOG CPLEX. http://www-01.ibm.com/software/integration/optimization/ cplex-optimizer/. Accessed 05 February 2012

22. T Rappaport, Wireless communications: principles and practice, 2nd edn. (Prentice Hall, Upper Saddle River, NJ, 2002)
23. P Gupta, PR Kumar, The capacity of wireless networks. IEEE Trans. Inf. Theory 46(2), 388-404 (2000)

24. MR Garey, DS Johnson, L Stockmeyer, Some simplified NP-complete graph problems. Theor Comput Sc 1, 237-267 (1976)

25. HS Wilf, Algorithms and complexity (Prentice Hall Inc, London, 1986)

26. R Jain, The Art of computer systems performance analysis: techniques for experimental design, measurement, simulation, and modeling (Wiley-Interscience, New York, 1991)

doi:10.1186/1687-1499-2013-229

Cite this article as: Chaudhry et al:: On the number of channels required for interference-free wireless mesh networks. EURASIP Journal on Wireless Communications and Networking 2013 2013:229.

\section{Submit your manuscript to a SpringerOpen ${ }^{\circ}$ journal and benefit from:}

- Convenient online submission

- Rigorous peer review

- Immediate publication on acceptance

- Open access: articles freely available online

- High visibility within the field

- Retaining the copyright to your article

Submit your next manuscript at $>$ springeropen.com 